\title{
Genetic landscape of metastatic and recurrent head and neck squamous cell carcinoma
}

\author{
Matthew L. Hedberg, ${ }^{1,2,3}$ Gerald Goh, ${ }^{4,5}$ Simion I. Chiosea, ${ }^{6}$ Julie E. Bauman, ${ }^{7}$ Maria L. Freilino, ${ }^{3}$ Yan Zeng, ${ }^{3}$ Lin Wang, ${ }^{6}$ \\ Brenda B. Diergaarde, ${ }^{8}$ William E. Gooding, ${ }^{9}$ Vivian W.Y. Lui, ${ }^{10}$ Roy S. Herbst, ${ }^{4}$ Richard P. Lifton, ${ }^{4,5}$ and Jennifer R. Grandis ${ }^{11}$ \\ 'Medical Scientist Training Program, University of Pittsburgh-Carnegie Mellon University, Pittsburgh, Pennsylvania, USA. ²Department of Pharmacology and Chemical Biology and \\ ${ }^{3}$ Department of Otolaryngology, University of Pittsburgh School of Medicine, Pittsburgh, Pennsylvania, USA. ${ }^{4}$ Department of Genetics, Yale University School of Medicine, New Haven, Connecticut, USA. \\ ${ }^{5}$ Howard Hughes Medical Institute, New Haven, Connecticut, USA. ${ }^{6}$ Department of Pathology and 'Department of Medicine, Division of Hematology/Oncology, University of Pittsburgh School of Medicine, \\ Pittsburgh, Pennsylvania, USA. ${ }^{8}$ Department of Epidemiology, Graduate School of Public Health, University of Pittsburgh, and University of Pittsburgh Cancer Institute, Pittsburgh, Pennsylvania, USA. \\ ${ }^{9}$ Biostatistics Facility, University of Pittsburgh Cancer Institute, Pittsburgh, Pennsylvania, USA. ${ }^{10}$ Department of Pharmacology and Pharmacy, Department of Biochemistry, Li-Ka Shing Faculty of Medicine, \\ University of Hong Kong, Pokfulam, Hong Kong. "'Department of Otolaryngology-Head and Neck Surgery, UCSF, San Francisco, California, USA.
}

BACKGROUND. Recurrence and/or metastasis occurs in more than half of patients with head and neck squamous cell carcinoma (HNSCC), and these events pose the greatest threats to long-term survival. We set out to identify genetic alterations that underlie recurrent/metastatic HNSCC.

METHODS. Whole-exome sequencing (WES) was performed on genomic DNA extracted from fresh-frozen whole blood and patient-matched tumor pairs from 13 HNSCC patients with synchronous lymph node metastases and 10 patients with metachronous recurrent tumors. Mutational concordance within and between tumor pairs was used to analyze the spatiotemporal evolution of HNSCC in individual patients and to identify potential therapeutic targets for functional evaluation.

RESULTS. Approximately $86 \%$ and $\mathbf{6 0} \%$ of single somatic nucleotide variants (SSNVs) identified in synchronous nodal metastases and metachronous recurrent tumors, respectively, were transmitted from the primary index tumor. Genes that were mutated in more than one metastatic or recurrent tumor, but not in the respective primary tumors, include C17orf104, inositol 1,4,5-trisphosphate receptor, type 3 (ITPR3), and discoidin domain receptor tyrosine kinase 2 (DDR2). Select DDR2 mutations have been shown to confer enhanced sensitivity to SRC-family kinase (SFK) inhibitors in other malignancies. Similarly, HNSCC cell lines harboring endogenous and engineered DDR2 mutations were more sensitive to the SFK inhibitor dasatinib than those with WT DDR2.

CONCLUSION. In this WES study of patient-matched tumor pairs in HNSCC, we found synchronous lymph node metastases to be genetically more similar to their paired index primary tumors than metachronous recurrent tumors. This study outlines a compendium of somatic mutations in primary, metastatic, and/or recurrent HNSCC cancers, with potential implications for precision medicine approaches.

FUNDING. National Cancer Institute, American Cancer Society, Agency for Science, Technology and Research of Singapore, and Gilead Sciences Inc.

\section{Introduction}

Head and neck squamous cell carcinoma (HNSCC) is the seventh most common incident cancer worldwide, with more than 600,000 new cases each year (1). The major risk factors for HNSCC are tobacco use, alcohol consumption, and/or infection with oncogenic strains of HPV, primarily HPV 16 (2). Despite advances in multimodality treatment, including surgery, radiation, and chemotherapy, 5-year overall survival has improved modestly

Authorship note: Matthew L. Hedberg and Gerald Goh contributed equally to this work. Conflict of interest: The authors have declared that no conflict of interest exists. Submitted: March 27, 2015; Accepted: October 22, 2015.

Reference information: J Clin Invest. 2016;126(1):169-180. doi:10.1172/JCI82066. over 3 decades, and this persistent mortality is largely due to high rates of regional metastasis and locoregional recurrence (3).

HNSCC metastases almost always arise first in the cervical lymph nodes (4). Most patients are diagnosed with locally advanced disease, and more than half have cervical lymph node metastases present at initial diagnosis (5). Clinically, this is classified as synchronous nodal metastasis and is an indicator of a poor prognosis. Five-year adjusted survival rates range from approximately $30 \%$ to $60 \%$ for patients with synchronous nodal metastasis compared with approximately $85 \%$ for patients whose cancer has not metastasized (6). Patients with synchronous nodal metastasis are also more likely to develop locoregional or distant metastatic recurrence of HNSCC after completing curative-intent therapy (7). 


\section{Table 1. Clinical characteristics of synchronous nodal metastasis cohort}

\begin{tabular}{lccccc}
\multicolumn{5}{c}{ Synchronous nodal metastasis group } \\
Index primary tumor
\end{tabular}

All index primary tumors and synchronous nodal metastases were surgically resected. Dx, diagnosis; site, anatomic location of primary tumor; path, pathological; OC, oral cavity; P, pharynx; L, larynx, LN, lymph node (cervical).

Rates of recurrence following treatment of an index HNSCC tumor range from approximately $25 \%$ to $50 \%$, depending on the anatomical location of the primary tumor, stage at diagnosis, and HPV status (8). Relapse after initial curative-intent treatment is known as metachronous recurrence. In patients who experience recurrence, approximately $25 \%$ to $50 \%$ will do so more than once (8). Recurrent tumors are more likely to be locoregional than distant (9). Median survival following metachronous recurrence is less than 22 months in patients who are eligible for salvage surgery or reirradiation and less than 12 months for those receiving palliative chemotherapy alone (8). Recurrence in HNSCC is often resistant to standard therapy and is generally considered incurable $(8,10)$.

The genetic alterations underlying nodal metastasis and recurrence are incompletely understood and present a fundamental challenge to the development of more effective therapies. Next-generation sequencing of several cancers has greatly expanded our appreciation of the genetic heterogeneity that exists in a variety of malignancies. Cumulative evidence implicates a complex, nonlinear, branched evolution model of subclonal populations within tumors that defines dynamic processes that likely mediate the expansion of minor subclones under the selective pressure of therapy, culminating in metachronous recurrences that are often treatment resistant $(11,12)$. In hematological malignancies, distinct patterns of clonal evolution in the development of therapeutic resistance and relapse have been reported $(13,14)$. In melanoma treated with MEK inhibitors, sequencing of recurrent tumors has identified activating mutations in MEK2 that confer resistance to targeted therapy (15). Whole-exome sequencing (WES) studies have revealed mutational signatures induced by temozolomide in recurrent glioma, demonstrated inherent functional variability in recurrent clones that affect response to chemotherapy in colorectal cancer, and shown that treatment can be guided by the sequencing of metastases or circulating tumor cells in breast cancer (16-19).

Next-generation sequencing studies have not yet characterized synchronous nodal metastasis or metachronous recurrence in HNSCC. Microarray-based expression profiling of unmatched normal mucosa, primary tumors, lymph node metastases, and recurrent HNSCC lesions identified mRNA expression signatures predictive of metastasis and recurrence, but the clinical impact of these observations remains unrealized (20-23). Only one study included analysis of patientmatched index primary tumors and synchronous nodal metastases; this found that the expression profiles of primary tumors were largely preserved in their respective metastatic lymph nodes (23). To date, WES studies in HNSCC have been conducted almost entirely in newly diagnosed primary tumors; these studies have enriched our understanding of the genetic alterations underlying the pathophysiology of primary HNSCC and identified novel therapeutic targets (24-31). Mutational compendiums in the setting of metastasis and/or recurrence may contribute to similar advances for these HNSCC patients with especially poor prognoses. In an effort to define and target the genetic alterations underlying metastasis and recurrence in HNSCC, we performed WES of patient-matched tumor pairs in the setting of synchronous nodal metastasis or metachronous recurrence.

\section{Results}

Patient characteristics and WES. The cohorts analyzed in this study were 2 groups of patients with HNSCC from the University of Pittsburgh: the synchronous nodal metastasis group and the metachronous recurrence group (Supplemental Figure 1; supplemental material available online with this article; doi:10.1172/JCI82066DS1). The synchronous nodal metastasis group consisted of 13 patients contributing blood, primary tumor, and synchronous nodal metastases. The metachronous recurrence group consisted of 10 patients contributing blood, primary tumor, and recurrent tumor. All patients were treated with curative intent for the index tumor, and all metachronous recurrences underwent salvage therapy.

Clinical and pathologic characteristics were typical of a surgically treated HNSCC population and are summarized in Tables 1 and 2. Briefly, all patients were of mixed European descent, $83 \%$ of the patients were male (19/23), mean age at diagnosis was 61.9 \pm 11.2 years (range: $44-79)$, 87\% (20/23) had a history of substantial tobacco exposure (generally $>20$ pack years), $74 \%(17 / 23)$ had documented alcohol use, and only 1 patient had $\mathrm{HPV}^{+}$disease. Of the 5 patients in the metachronous recurrence group with nodal disease at the time of initial diagnosis, all had evidence of extracapsular spread as did 10 of the 13 patients in the synchronous nodal metastasis group.

The majority of the index primary tumors were located in the oral cavity $(61 \% ; n=14)$, with the remainder distributed between the larynx $(22 \% ; n=5)$ and pharynx $(17 \% ; n=4)$. As is commonly seen in HNSCC, most patients presented with advanced disease. Of the newly diagnosed index primary tumors, $25 \%$ and $70 \%$ were American Joint Committee on Cancer (AJCC) stage III or 
Table 2. Clinical characteristics of metachronous recurrence cohort.

\begin{tabular}{|c|c|c|c|c|c|c|c|c|}
\hline \multicolumn{9}{|c|}{ Metachronous recurrence group } \\
\hline \multicolumn{3}{|c|}{ General characteristics } & \multicolumn{4}{|c|}{ Index primary tumor } & \multicolumn{2}{|c|}{$\begin{array}{l}\text { Metachronous } \\
\text { recurrent tumor }\end{array}$} \\
\hline Patient ID \# & Sex & Age at Dx (yr) & Site & Path. stage & AJCC stage & Adjuvant treatment & $\begin{array}{c}\text { Time to } \\
\text { recurrence (mo) }\end{array}$ & Site \\
\hline PY-1 & M & 78 & $O C$ & T3 N1 MO & III & None & 2.3 & $P$ \\
\hline PY-3 & $\mathrm{F}$ & 67 & $\mathrm{OC}$ & T2 N2B MO & IVA & RT & 6.3 & $O C$ \\
\hline PY-4 & M & 44 & T4A N2B MO & IVA & RT, Cisplatin, and Vectibix & 6.8 & $P$ & \\
\hline PY-6 & M & 52 & $P$ & TX NX MX & NA & IMRT, cetuximab, and pemetrexed & 10.5 & $\mathrm{LN}$ \\
\hline PY-7 & M & 49 & L & T4A N1 MO & IVA & None & 2.9 & $\mathrm{LN}$ \\
\hline PY-8 & M & 76 & $\mathrm{OC}$ & TX NX MX & NA & RT & 33.9 & $\mathrm{P}$ \\
\hline PY-9 & M & 60 & $O C$ & T2 NO MX & $\|$ & None & 4.7 & $\mathrm{LN}$ \\
\hline PY-10 & $M$ & 58 & $\mathrm{OC}$ & TX NX MX & NA & None & 2.2 & $O C$ \\
\hline PY-11 & $M$ & 74 & $\mathrm{OC}$ & T3 N2C MO & IVA & RT and Cisplatin & 9.7 & $O C$ \\
\hline
\end{tabular}

All index primary tumors and metachronous recurrences were surgically resected. IMRT, intensity-modulated radiation therapy; RT, radiation therapy. Index primary tumors for patients PY-6, PY-8, and PY-10 were recurrences, which are not staged.

IVA, respectively; metachronous recurrent tumors are not staged (Table 2 and ref. 32). All metachronous recurrences sequenced in this study were locoregional and, compared with their paired index primary tumors, 7/10 were located in different anatomic subsites of the head and neck (Table 2). Median time to recurrence in the metachronous recurrence group was 5.5 months (range, 2.2-33.9) (Table 2). Of 10 patients in the metachronous recurrence group, 2 received adjuvant radiation therapy and 3 received adjuvant chemotherapy in combination with radiation after resection of the index primary tumor (Table 2).

Genomic DNA from patient-matched tumor pairs and peripheral blood mononuclear cells (PBMCs) was subjected to exome sequencing as described in Methods. By design, tumors were sequenced to higher depth of coverage than normal samples (PBMCs). Normal samples were sequenced to a mean depth of 123 independent reads per targeted base and tumors to a mean of 202 independent reads (Supplemental Table 1). Single somatic nucleotide variants (SSNVs) and somatic copy number variants (SCNVs) were called (see Methods), and tumor purity was estimated from deviation in minor allele frequency of heterozygous SSNVs in segments showing loss of heterozygosity (LOH) (Supplemental Table 2). In 1 primary tumor, 2 synchronous metastases, and 1 metachronous recurrence, estimated tumor purity was too low to yield highquality sequencing data (Supplemental Table 2). These patients were not included in the analysis of genetic concordance between matched tumor pairs, but the tumors obtained from them that did yield high-quality sequencing data were included when reporting overall mutation rates in each tumor type (11 total synchronous metastases and 9 total metachronous recurrences). All SSNVs identified by exome sequencing are enumerated in Supplemental Table 10, and sequencing files were deposited in the NCBI's database of Genotypes and Phenotypes (dbGaP phs0001007.v1.p1).

Genetic profiles of index primary tumors. Among 22 primary tumors, 1,961 SSNVs were identified in 1,666 genes (Supplemental Table 10). Tumors averaged 89.1 SSNVs (range 3-413), and the ratio of nonsynonymous/synonymous variants in coding regions was 3.14:1. Analysis of mutation burden with MutSigCV (http://www.broadinstitute.org/cancer/software/genepattern/ modules/docs/MutSigCV) revealed a single gene, tumor protein p53 (TP53), with a significant excess of SSNVs (mutated in 13 tumors, $\left.q=2.66 \times 10^{-6}\right)(33)$. We utilized the findings of Vogelstein et al. $(n=124)$, the The Cancer Genome Atlas (TCGA) PanCancer effort $(n=127)$, and the TCGA HNSCC cohort $(n=11)$ to generate a composite list of 191 genes, which have either been established as cancer driver genes and/or as genes that are commonly mutated in HNSCC (cancer driver genes) (Supplemental Table 3 and refs. 30, 34, 35). Forty-seven nonsynonymous SSNVs were identified in 28/191 cancer driver genes, which is significantly greater than expected by chance $\left(P=5.2 \times 10^{-5}\right.$, Monte Carlo) (Supplemental Table 4).

GISTIC2 analysis of SCNVs in the primary tumors of our cohort identified 8 significantly amplified regions, including portions of $3 \mathrm{q}$ and $8 \mathrm{q}$ that were amplified in a majority of tumors in the TCGA HNSCC cohort as well as $3 \mathrm{q} 26.32\left(q=4.95 \times 10^{-6}\right)$, $7 \mathrm{p} 11.2\left(q=2.62 \times 10^{-3}\right)$, and $11 \mathrm{q} 13.3\left(q=1.14 \times 10^{-3}\right)$, which were also significantly amplified in the TCGA HNSCC cohort and overlap well-known cancer driver genes phosphatidylinositol-4,5-bisphosphate 3-kinase, catalytic subunit alpha (PIK3CA), EGFR, and cyclin D1 (CCND1), respectively (Supplemental Figure 2 and refs. $30,36)$. We also observed 8 segments with significant recurrent somatic deletions, including portions of $3 p$ and $8 p$ that were lost in a majority of tumors in the TCGA HNSCC cohort as well as 9p21.3 ( $q=0.15)$, which was also significantly deleted in the TCGA HNSCC cohort and overlaps cyclin-dependent kinase inhibitor 2A (CDKN2A) and CDKN2B (Supplemental Figure 2 and refs. 30, 36). Overall, the genetic profiles of the index primary tumors in this cohort are similar to those reported in other WES studies of primary HNSCC (24-31).

Index primary tumors from patients in the synchronous nodal metastasis group had a significantly greater mutational burden than those from patients in the metachronous recurrence group $\left(125.9 \pm 103.2\right.$ versus $36.0 \pm 31.6 \mathrm{SSNVs}, P=9.94 \times 10^{-3}$, 2 -tailed 



Figure 1. SSNVs in HNSCC tumors. (A) Top panel: distribution of SSNVs in each tumor pair; synchronous nodal metastases on the left, metachronous recurrences on the right. Lower panel: panels describing presence of SSNVs in select cancer driver genes in each tumor pair. SSNVs were identified as unique to primary tumor (dark blue), shared between paired tumors (light blue), or unique to metastasis or recurrent tumor (gold). (B) Differences in subclonal architecture in the primary and synchronous nodal metastatic tumors from patient PY- $16.44 \%$ of SSNVs were private to the metastatic tumor (clusters 4 and 5) and 25\% private to the primary tumor (cluster 1). (C) SSNVs in the samples from patient PY-8 are unique to the primary tumor (cluster 1 ) or the recurrent tumor (cluster 2); shared SSNVs (clusters 3 and 4) are germline. VAF, variant allele frequency.

$t$ test). Even after excluding PY-15 (413 SSNVs) as an outlier, there was a significant difference in the number of SSNVs between the 2 groups $\left(102.0 \pm 59.2\right.$ versus $36.0 \pm 31.6, P=4.21 \times 10^{-3}$, 2 -tailed $t$ test). Similarly, SCNV burden in the index primary tumors differed significantly, with a higher fraction of the genome in index primary tumors from the synchronous nodal metastasis group harboring SCNVs than those in the metachronous recurrence group (Supplemental Table 5, $0.36 \pm 0.13$ versus $0.19 \pm 0.13, n=13$ and $n=9, P=0.017$, Mann-Whitney $U$ test).

Genetic profiles of synchronous nodal metastases. In the synchronous nodal metastasis group, we obtained high-quality sequencing data from the matched PBMCs, primary tumor, and synchronous nodal metastases of 11 patients, allowing for the analysis of genetic concordance within matched tumor pairs in these patients (Figure 1A and Supplemental Table 2). The nodal metastases in these patients harbored 1,499 SSNVs in 1,310 genes (Figure 1A and Supplemental Table 10). Thirty-three nonsynonymous SSNVs in the metastases were found in 23/191 cancer driver genes, which is significantly greater than expected by chance $(P=0.0013$, Monte Carlo) (Supplemental Table 6 and refs. 30, 34, $35)$. In the primary tumors of these 11 patients, $84.8 \%(979 / 1154)$ of nonsynonymous SSNVs and $94.1 \%$ (32/34) of nonsynonymous SSNVs in cancer driver genes were transmitted to the nodal metas- tases (not a significantly different rate of transmission, $P=0.15$, Fisher test) (Figure 1A and Supplemental Table 10). In the nodal metastases of these 11 patients, 13.9\% (208/1499) of the SSNVs were identified as newly arisen, i.e., absent in the primary tumor (see Methods), including 1 new mutation in a cancer driver gene, $M L L 4$, in PY-15 (Figure 1A, Supplemental Table 10, and Supplemental Table 6).

The PyClone algorithm was used to identify and quantify clonal populations in the primary and metastatic tumors (37). In 10 of 11 patients, $80 \%$ or more of the SSNVs identified in the nodal metastases were transmitted from the primary tumor (Supplemental Table 10). In those 10 patients, PyClone analysis revealed that the majority of SSNVs were transmitted in a stable fashion, with the primary clone (containing $>50 \%$ of SSNVs) being present at similar cellular prevalence in both the primary and metastatic tumors (Supplemental Table 7). In the 11th patient, PY-16, 44\% of SSNVs were unique to the metastatic tumor (clusters 4 and 5 in Figure 1B), which had lost $25.2 \%$ of the SSNVs found in the primary tumor (cluster 1 in Figure 1B).

GISTIC2 analysis of SCNVs in the synchronous nodal metastases revealed 4 regions of significant focal amplification and 4 regions of significant focal deletion, with copy number gains encompassing EGFR, and CCND1 and copy number losses encom- 


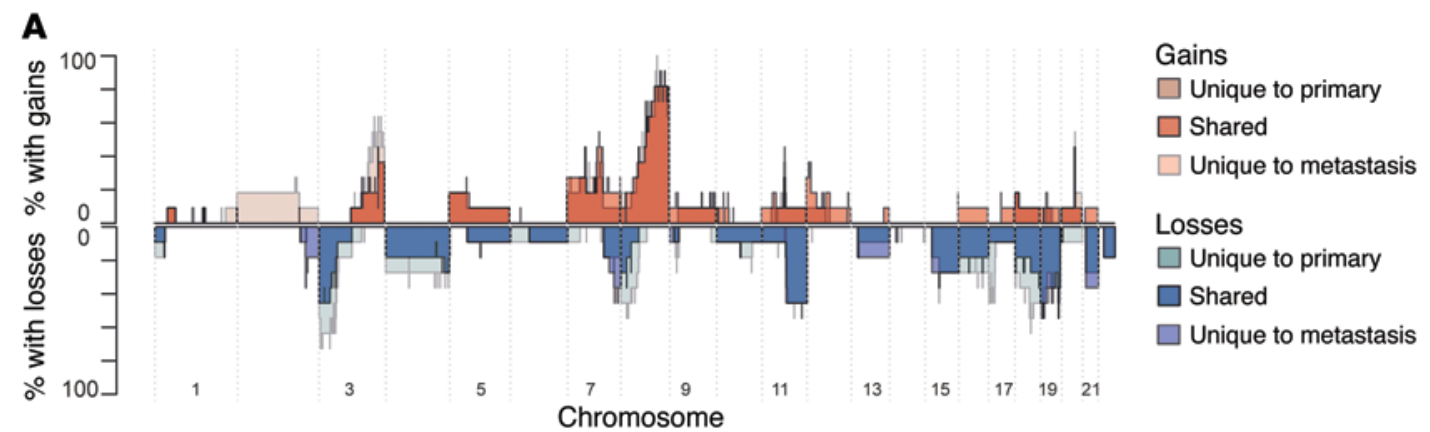

B

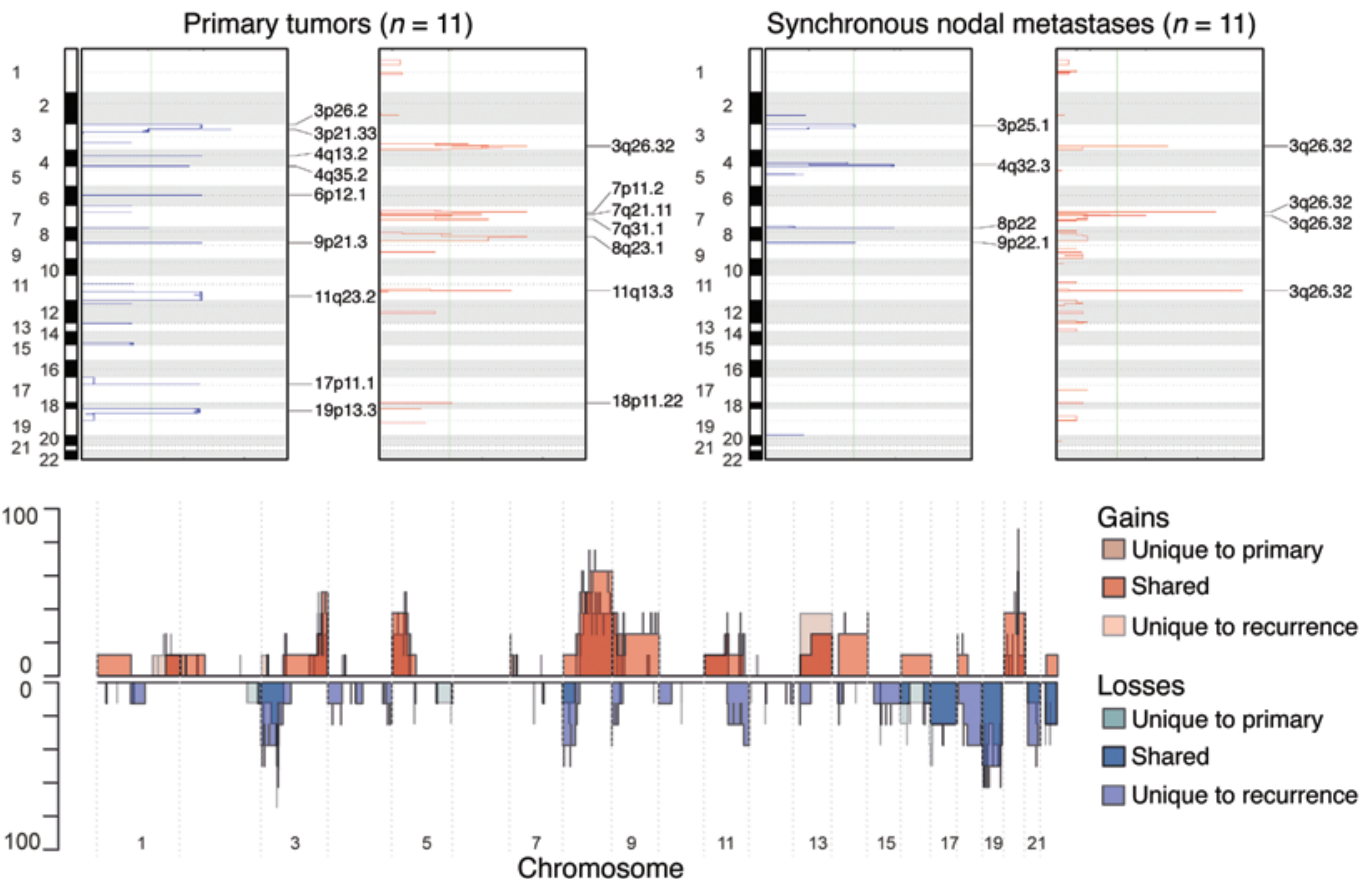

D
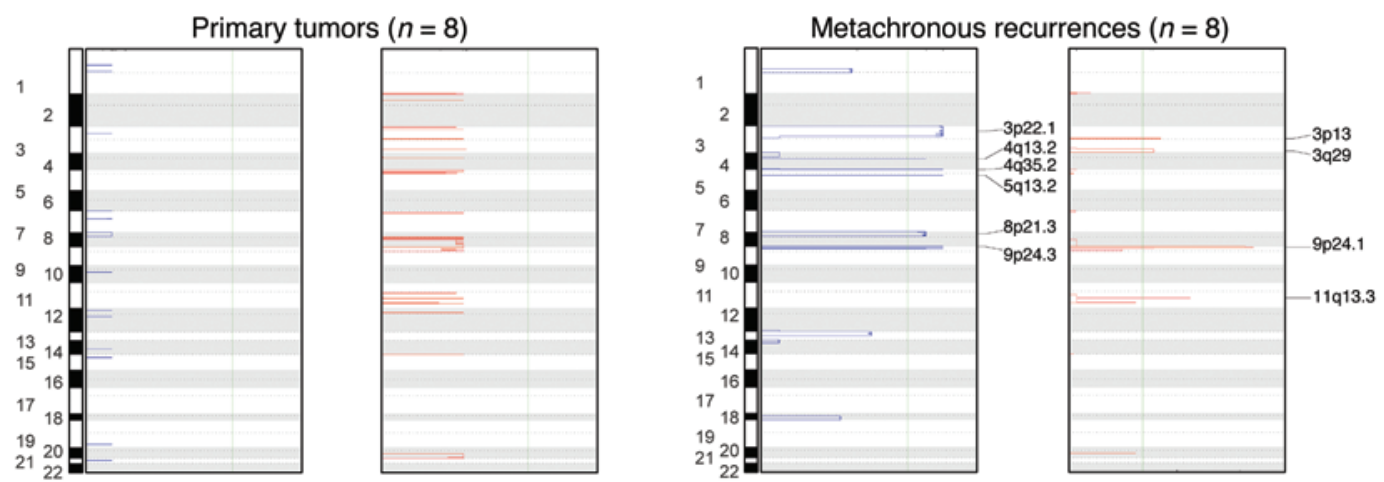

Figure 2. Somatic CNVs in HNSCC tumors. (A) Overview of CNVs and (B) GISTIC significant peaks across 11 primary tumors and paired synchronous nodal metastases. (C) Overview of CNVs and (D) GISTIC significant peaks across 8 primary tumors and paired metachronous recurrences. Red, amplifications; blue, deletions; shades of red and blue, unique to primary or metastatic/recurrent tumor or transmitted between tumors; green line, $q=-.25$, signifying threshold for significance.

passing $C D K N 2 A$ and $C D K N 2 B$ (Supplemental Figure 3 and ref. 36). The copy number profiles between matched primary tumors and synchronous nodal metastases were not significantly different (fraction of genome-harboring SCNVs, $0.38 \pm 0.14$ versus 0.32 $\pm 0.14, n=11, P=0.48$, Mann-Whitney $U$ test, Supplemental Table 5 and Figure 2A). Many of the significant SCNV peaks identified in the nodal metastases of these patients arose in the primary tumor; $3 / 4$ regions of significant focal amplification identified in the nodal metastases were also significantly amplified in the primary tumors (3q26.32, 7p11.2, and 11q13.3) (Figure 2B).

Among these 11 patients, 60 genes harbored nonsynonymous mutations in 2 or more patients (Figure 1A and Supplemental Table 10). Three of those 60 genes, TP53, FAT1, and MLL2, are cancer driver genes. Two genes were newly mutated in the synchronous metastases of 2 patients, C17orf104 and inositol 1,4,5-trisphosphate receptor, type 3 (ITPR3) $(P=0.37$, Monte Carlo) 
Table 3. Genes that are exclusively mutated in $\mathbf{2}$ synchronous nodal metastases

\begin{tabular}{|c|c|c|c|c|c|c|c|c|}
\hline \multirow[t]{2}{*}{ Gene } & \multirow[t]{2}{*}{ Patient } & \multirow[t]{2}{*}{ aa change } & \multicolumn{3}{|c|}{ Index primary } & \multicolumn{3}{|c|}{ Synchronous nodal metastasis } \\
\hline & & & Ref & Nonref & MAF & Ref & Nonref & MAF \\
\hline \multirow[t]{2}{*}{ C17orf104 } & PY-16 & $\mathrm{H} 846 \mathrm{Y}$ & 217 & 0 & 0 & 176 & 56 & 0.241 \\
\hline & PY-21 & Q145 ${ }^{\mathrm{A}}$ & 234 & 0 & 0 & 215 & 34 & 0.137 \\
\hline \multirow[t]{2}{*}{ ITPR3 } & PY-15 & R149L & 44 & 0 & 0 & 55 & 32 & 0.368 \\
\hline & PY-16 & R64H & 164 & 0 & 0 & 137 & 31 & 0.185 \\
\hline
\end{tabular}

Ref, number of reference sequence reads; nonref, number of nonreference sequence reads; MAF, minor allele frequency (adjusted for estimated tumor purity). ${ }^{A}$ Nonsense mutation.

(Table 3). The precise function or functions of C17orf104 have not been described, whereas ITPR3 encodes a calcium channel receptor that binds inositol 1,4,5-trisphosphate, mediates intracellular calcium levels, and is important to exocrine functions that influence metabolism and growth $(38,39)$. ITPR3 has been implicated in breast cancer proliferation, and elevated expression levels correlate with increased invasion and metastasis and decreased survival in colorectal cancer as well as dissemination of gastric cancers (40-42).

Genetic profiles of metachronous recurrent tumors. In the metachronous recurrence group, we obtained high-quality sequencing data from the matched PBMCs, primary tumor, and metachronous recurrent tumors of 8 patients, allowing for the analysis of genetic concordance in matched tumor pairs from these patients (Figure 1A and Supplemental Table 2). The metachronous recurrent tumors in these 8 patients harbored 457 SSNVs in 441 genes. Nine nonsynonymous SSNVs were found in 6/191 cancer driver genes, this number being significantly greater than expected by chance ( $P=0.019$, Monte Carlo) (Supplemental Table 9 and refs. 30, 34, $35)$. In the primary tumors from these 8 patients, $90.1 \%(274 / 304)$ of SSNVs overall and $100 \%(5 / 5)$ of SSNVs in cancer driver genes were transmitted to the metachronous recurrent tumors (Figure 1A). In the metachronous recurrences of these 8 patients, $40.0 \%$ $(183 / 457)$ of SSNVs were identified as newly arisen (Figure 1A). The copy number profiles between matched primary tumors and metachronous recurrent tumors were not significantly different (fraction of genome harboring SCNVs, $0.21 \pm 0.13$ versus $0.34 \pm 0.12$, $P=0.05, n=8$, Mann-Whitney $U$ test, Supplemental Table 5 and Figure 2C). GISTIC2 analysis of these 8 primary tumor-metachronous recurrence pairs identified no regions of significant focal amplification or deletion in the primary tumors, whereas 4 and 6 regions of significant focal amplification and deletion were observed in the recurrent tumors, respectively, with copy number gains overlapping PIK3CA, NFIB, and CCND1 and copy number losses overlapping TATDN2 and FAT1 (Figure 2D, Supplemental Figure 5, and ref. 36).

The PyClone algorithm was used to determine whether the metachronous recurrent tumors were largely clonal, as was observed in the synchronous nodal metastases (37). The total mutation load in the tumor pairs from patients PY-3, PY-6, PY-7, and PY-10 was too low (<30 SSNVs per pair) for analysis with this technique. In the metachronous recurrent tumors from patients PY-1, PY-5, and PY-11, 90\% or more of the SSNVs identified were transmitted from the primary index tumor and present at similar cellular prevalence (Supplemental Table 7). In the metachronous recurrent tumor from patient PY-4, 60.2\% of the SSNVs identified were transmitted from the primary index tumor, and the recurrence was composed of a set of SSNVs seeded from the primary tumor, present at high cellular prevalence (0.9-1.0), and a set of SSNVs unique to the metachronous recurrent tumor (40\%), present at a moderate cellular frequency (a subclonal population) (Supplemental Table 10 and Supplemental Figure 4). In patient PY-8, the metachronous recurrent tumor was genetically distinct from the index primary tumor, with 110 and 26 SSNVs unique to the metachronous recurrent and index primary tumors, respectively (clusters 1 and 2); only 3 SNVs, all of which were also detected at trace levels in the matched normal tissue, were shared between the 2 tumors (clusters 3 and 4 ), demonstrating that this is a true "second primary" or "second field" tumor (SPT) (Supplemental Table 10 and Figure 1C). Also, newly arisen mutations were identified in 4 cancer driver genes of this SPT: KDM5C, POLQ SF3B1, and TP53 (Supplemental Table 8).

Table 4. DDR2 is exclusively mutated in $\mathbf{2}$ metachronous HNSCC recurrences

\begin{tabular}{|c|c|c|c|c|c|c|c|c|}
\hline \multirow[t]{2}{*}{ Gene } & \multirow[t]{2}{*}{ Patient } & \multirow[t]{2}{*}{ aa change } & \multicolumn{3}{|c|}{ Index primary } & \multicolumn{3}{|c|}{ Metachronous recurrence } \\
\hline & & & Ref & Nonref & MAF & Ref & Nonref & MAF \\
\hline \multirow[t]{2}{*}{$D D R 2$} & PY-3 & p.R709A & 77 & 0 & 0 & 91 & 16 & 0.15 \\
\hline & PY-8 & p.1474M & 190 & 0 & 0 & 141 & 50 & 0.262 \\
\hline \multirow[t]{2}{*}{ Gene } & Patient & aa change & \multicolumn{3}{|c|}{ Index primary } & \multicolumn{3}{|c|}{ Synchronous nodal metastasis } \\
\hline & & & Ref & Nonref & MAF & Ref & Nonref & MAF \\
\hline$D D R 2$ & PY-22 & p. $1724 M$ & 104 & 74 & 0.42 & 111 & 50 & 0.31 \\
\hline
\end{tabular}

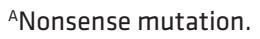




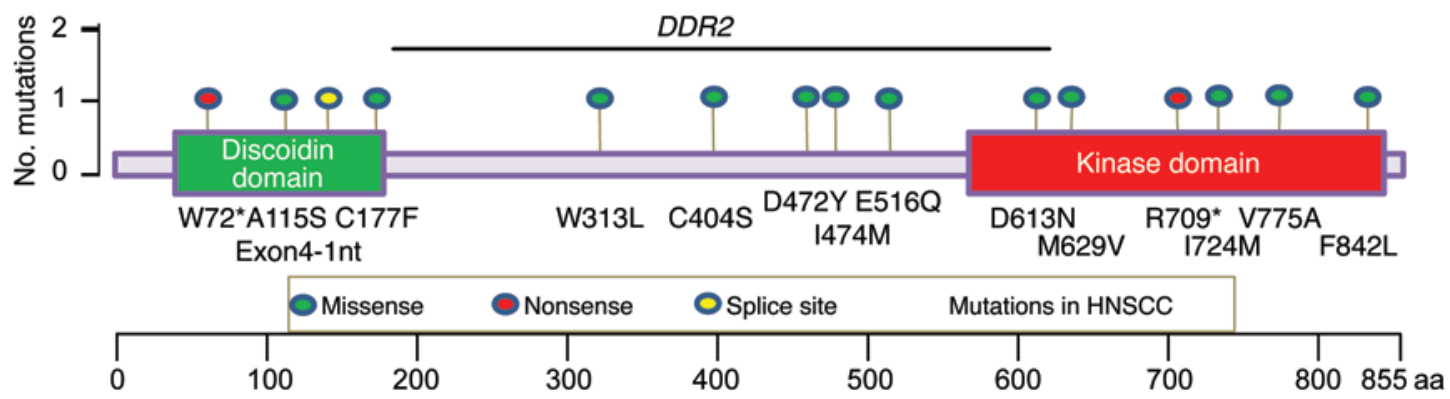

Figure 3. DDR2 mutations in HNSCC. The genetic alterations in DDR2 identified in our cohort and the previously reported, transcript-altering DDR2 mutations identified in other sequencing studies of primary HNSCC tumors are displayed. $2 / 9(22.2 \%)$ metachronous recurrent tumors sequenced in this study had newly arisen DDR2 mutations vs. 12/658 (1.8\%) newly diagnosed primary HNSCC tumors in other studies.

Among the 8 patients with high-quality sequencing data from all 3 tissue samples, 4 genes, discoidin domain receptor tyrosine kinase 2 (DDR2), OR7A5, SYNE1, and TP53, harbored nonsynonymous mutations in 2 or more patients (Supplemental Table 10). $D D R 2$ was the only gene with newly acquired mutations in the metachronous tumors of 2 patients, PY-3 and PY- 8 (Table 4 and Figures 3 and 4). DDR2 encodes the discoidin domain receptor 2, which is a collagen-stimulated receptor tyrosine kinase implicated in a wide range of processes including regulation of epithelialto-mesenchymal transition (EMT) and osteogenic/chondrogenic differentiation $(43,44)$. Although the functional mechanisms of $D D R 2$ expression and/or mutation in disease are incompletely understood, alterations in DDR2 have been implicated in cellular adhesion, migration, invasion, and metastasis in prostate cancer, breast cancer, and recently, HNSCC (45-48). In total, DDR2 mutations were observed in 3 of the 19 patients whose tumor pairs had reliable WES data (Table 4). A DDR2(I724M) mutation was identified in both the index primary tumor and synchronous nodal metastasis of patient PY-22.

Patients PY-3 and PY-8 both developed metachronous recurrences within the field of radiation therapy following resection of their index primary tumors. DDR2(R709*) and DDR2(I474M) mutations were identified in their metachronous recurrent tumors, respectively, and appeared to be newly arisen, as they were not identified in the paired index primary tumors from these patients. However, the depth of sequencing achieved in this study was not sufficient to rule out the possibility that these mutations were present in rare subclones in the primary tumors. The spec-
A

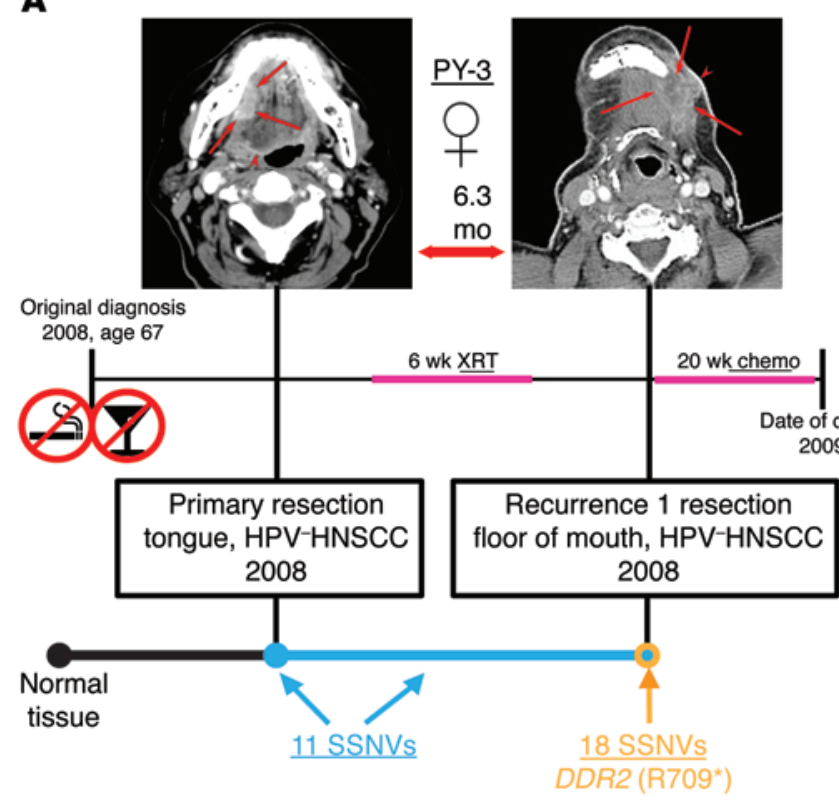

Germline/unknown
B
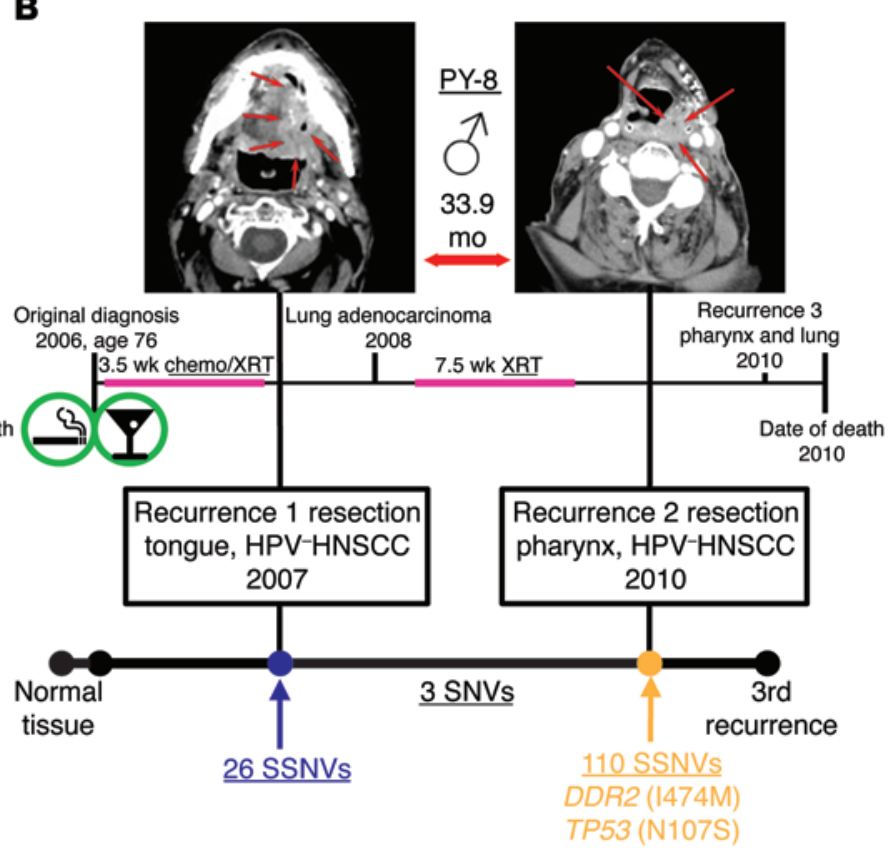

Shared Unique to metachronous recurrence

Figure 4. Spatial and temporal evolution of disease in patients harboring newly arisen DDR2 mutations in their recurrent tumors. The clinical histories of patient PY-3 (A) and patient PY-8 (B) are shown. Index primary and metachronous recurrent tumors assessed via WES are outlined and presented with CT imaging of the respective tumors. Treatments between cancer events are illustrated on the time line, and the time to recurrence between sequenced tumors is indicated in months. Below each time line is the mutational burden of each sequenced tumor for PY-3 and PY-8, respectively. SSNVs are defined as germline/unknown (black), unique to index primary tumor (dark blue), shared between paired tumors (light blue), or unique to metachronous recurrent tumor (gold). XRT, radiation therapy; chemo, chemotherapy. 
A

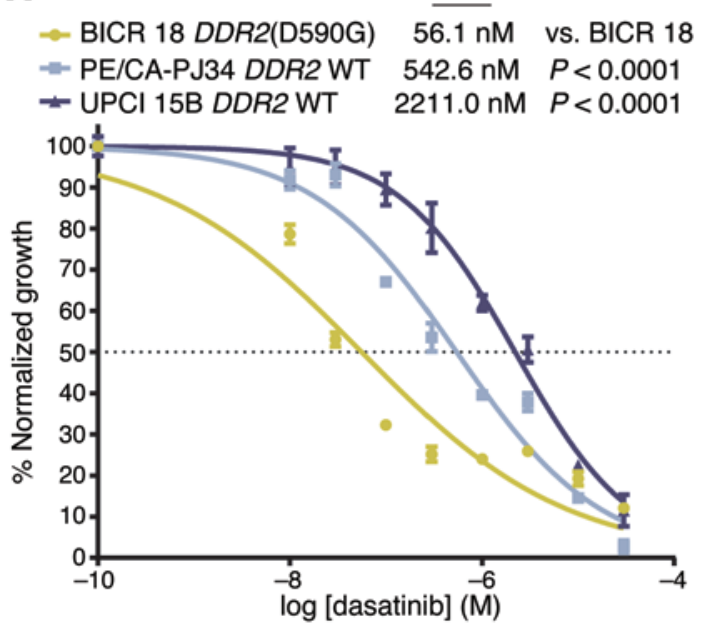

C

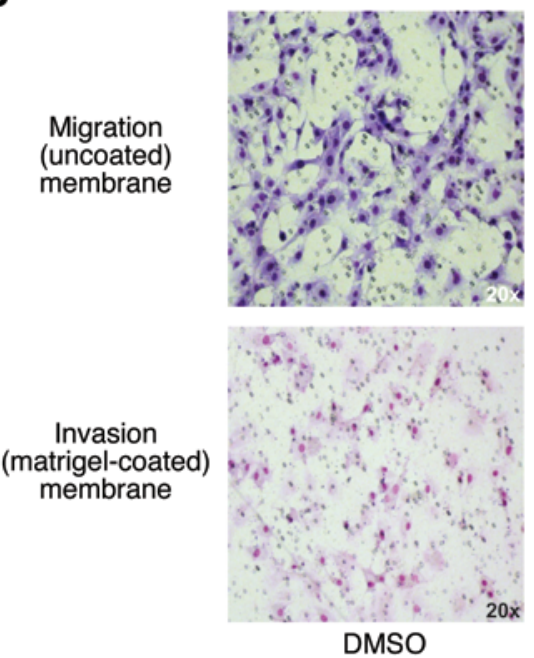

IC50
B


$10 \mathrm{nM}$
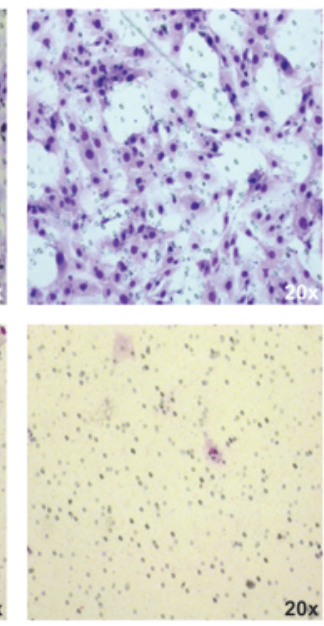

$50 \mathrm{nM}$

[dasatinib]

trum of DDR2 mutations in human HNSCC, including the new mutations reported here, is shown in Figure 3 and refs. 24-31. Figure 4 summarizes the clinical history of the 2 patients (PY-3 and PY-8) whose recurrent tumors were found to harbor new DDR2 mutations. Several DDR2 mutations, identified in squamous cell carcinoma of the lung, have been shown to be oncogenic and/or to confer high sensitivity to SRC-family kinase (SFK) inhibitors both in vitro and in vivo $(49,50)$. DDR2 mutations in recurrent/metastatic HNSCC could have important clinical implications, as significant clinical response to the SFK inhibitor, dasatinib, has been reported in 2 patients with squamous cell carcinoma of the lung harboring DDR2(S768R) mutations $(49,51)$.

HNSCC cell lines with DDR 2 mutations are sensitive to dasatinib. To investigate whether DDR2 mutations might serve as predictive biomarkers of dasatinib sensitivity in HNSCC, we treated a panel of HNSCC cell lines with dasatinib. BICR 18 harbors an endogenous DDR2(D590G) mutation, whereas PE/CA-PJ34 (clone C12) is known to have WT $D D R 2$, and UPCI 15B also has WT DDR2, by Sanger sequencing (data not shown).(52) By MTT assay, following 48 hours of dasatinib treatment, these 3 cell lines were found to have $\mathrm{IC}_{50}$ values of $5.608 \times 10^{-8} \mathrm{M}, 5.426 \times 10^{-7} \mathrm{M}$, and $2.211 \times$ $10^{-6} \mathrm{M}$, respectively (Figure $5 \mathrm{~A}$ ). In addition to its potent cytotoxic effect on BICR 18 cells at 48 hours, administration of dasatinib in the setting of 24-hour Matrigel invasion assays significantly inhibited the invasion of BICR 18 cells in a dose-dependent fashion (Figure 5, B and C). The sensitivity of BICR 18 cells to dasatinib was attenuated to a statistically significant degree in the setting of DDR2 knockdown, implicating DDR2 in the marked sensitivity of this mutant HNSCC cell line to dasatinib (Supplemental Figure 6). Similarly, when the most dasatinib-resistant HNSCC cell line from our panel, UPCI 15B, was engineered to express the DDR2 (R709*) mutation, identified in the metachronous recurrent tumor of patient PY-3, statistically significant sensitization to dasatinib treatment compared with EGFP control and WT DDR2 was observed (Supplemental Figure 7). Taken together, these data suggest that $D D R 2$ status may serve as a biomarker for response to SFK inhibitors in HNSCC.

\section{Discussion}

WES of patient-matched tumor pairs from 23 individuals with HNSCC demonstrates the intertumor genetic heterogeneity of this cancer. To our knowledge, this is the first study of HNSCC to examine intertumor genetic heterogeneity in synchronous nodal metastases and metachronous recurrence across multiple 
patients; it provides a model to determine patterns of mutation and clonal evolution that may be targetable for the treatment of recurrent/metastatic disease.

Primary tumors in our study averaged 67.6 nonsynonymous SSNVs per tumor, including mutations in tumor suppressor genes, oncogenes, and genes that have previously been found to be significantly mutated in HNSCC, such as AJUBA, CASP8, FAT1, FBXW7, NFE2L2, and TP53 (Supplemental Table 10 and Supplemental Table 4). Interestingly, primary tumors were not found to harbor mutations in several tumor suppressor genes and oncogenes previously implicated in HNSCC, including CCND1, PIK3CA, NOTCH1, PTEN, CDKN2A, HRAS, and EGFR. However, copy number alterations, consistent with previously published reports, were seen in several of these genes, and the overall genetic profiles of primary tumors in our study appear similar to those reported in other WES studies of primary HNSCC tumors (Supplemental Figure 2 and refs. 24-31).

Approximately 86\% (1290/1499) of the SSNVs identified in synchronous nodal metastases from 11 patient-matched tumor pairs were transmitted from their respective index primary tumors. This high degree of mutational similarity between primary tumors and synchronous nodal metastases in individual patients was also seen in the only other published sequencing report of patient-matched HNSCC tumors to date, in which whole genome sequencing was performed on 3 regions of a primary $\mathrm{HPV}^{+} \mathrm{HNSCC}$ tumor and 2 regions of a single synchronous cervical lymph node metastasis from 1 patient (53). In that study, Zhang et al. used multiple samples of the same tumor to study intratumor genetic heterogeneity and approximated the phylogenetic evolution and time line of cancer development in that patient. They estimated that the clones making up the nodal metastasis evolved at a significantly later point in time than the clones of the index primary tumor (53). We are unable to estimate the time line of clonal evolution, as our study design does not allow for the analysis of intratumor genetic heterogeneity. However, if metastatic potential is acquired in later stages of HNSCC progression, as posited by Zhang et al., then mutated genes identified in multiple nodal metastases, but not in the primary tumors, may confer metastatic ability. Newly arisen mutations in C17orf104 and ITPR3 were seen in the nodal metastases of 2 patients in the synchronous nodal metastases group; these represent plausible targets for investigation in future studies (Table 3). Alternatively, if the ability to metastasize is developed early in HNSCC development, as is the commonly held clinical belief and may be suggested by the observation that in $8 / 11$ patient-matched tumor pairs in the synchronous metastasis group, fewer SSNVs were identified in the metastases than in their paired primary tumors, then commonly transmitted mutations may confer metastatic ability (Supplemental Table 10).

Approximately 60\% (274/457) of the SSNVs identified in metachronous recurrences from 8 patient-matched tumor pairs were transmitted from their respective index primary tumors. This finding suggests a greater degree of intertumor genetic heterogeneity in the setting of metachronous recurrence versus synchronous nodal metastasis in HNSCC. This was largely expected given that, compared with their matched index primary tumors, metachronous recurrent tumors sequenced in this study were malig- nancies that arose in different anatomical microenvironments at later points in time, often following genotoxic therapy, including radiation and/or chemotherapy.

The field cancerization theory suggests that there are numerous malignant and premalignant fields present in the mucosa of HNSCC patients (54). This can lead to a high degree of intertumor genetic heterogeneity in the setting of recurrent disease, as metachronous tumors can be recurrences that evolved from cells in the initial primary tumor that survived treatment or they may arise from malignant clones that developed in a field unrelated to the initial cancer event, known as an SPT (3). To date, the differentiation of recurrence and SPT have been based on histologic appearance, anatomic location, and kinetics of tumor formation, with earlier events ( $<24$ months) generally considered to represent recurrences. Molecular diagnostic studies using mutated TP53 and patterns of genetic changes as markers suggest that as many as $50 \%$ of recurrent HNSCC tumors that arise within 3 years of initial treatment and occur within $2 \mathrm{~cm}$ of the original index tumor location may in fact be SPTs (9). In patient PY-8, we conclusively demonstrate an instance in which the metachronous tumor represents a true SPT. The metachronous tumor was found to harbor newly arisen mutations in multiple cancer driver genes and shared no SSNVs with the index primary tumor studied from that patient, apart from $3 \mathrm{SNVs}$ of uncertain, potentially germline, origin. Identification of the unique mutational profiles in recurrences and SPTs will be vital to the rational design of personalized therapy that appropriately targets the newly aberrant cellular processes contributing to the formation of metachronous head and neck tumors.

Although this cohort is small, it is striking that 2/9 metachronous recurrent tumors, including one true SPT, harbored DDR2 mutations that were not identified in their matched primary tumors. Sequencing data, utilizing platforms sufficient for detection of DDR2 mutations, are available for at least 658 newly diagnosed primary HNSCC tumors to date, and only 12 have been found to harbor transcript-altering DDR2 mutations (Figure 3 and refs. 24-31, 53, 55). The HNSCC cell line BICR 18, which harbors an endogenous DDR2 mutation, HNSCC cells engineered to express the $D D R 2$ mutations identified in metachronous recurrent HNSCC tumors, and DDR2 mutant tumors in 2 lung cancer case reports, demonstrated increased sensitivity to dasatinib (Figure 5, Supplemental Figure 7, and refs. 49, 50). While the precise mechanism or mechanisms underlying dasatinib sensitivity are beyond the scope of this report, the general mechanisms and consequences of discoidin domain receptor signaling in cancer are areas of ongoing research $(47-50,56)$. The DDR2(R709*) mutation identified in the metachronous recurrent tumor of patient PY-3, which conferred the greatest degree of sensitization to dasatinib in the present study, has also been reported in gastric and endometrial cancers. It is among the most common recurrent mutations and is the only recurrent truncation mutation, identified in DDR 2 across all cancers in the TCGA and COSMIC databases (57-60). Patient PY-3 was HPV negative, had no history of smoking or significant alcohol use, and had a low mutational burden, further implicating $D D R 2$ as a potential driver in the metachronous recurrence.

If validated in larger cohorts, our observation that $D D R 2$ mutations may be enriched in metachronous recurrences and may confer enhanced sensitivity to SFK inhibitors has important implica- 
tions for the treatment of recurrent disease, especially in light of the exceptional clinical response to dasatinib reported in 2 patients with lung squamous cell carcinoma harboring DDR2(S768R) mutations. As such, further mechanistic investigations to define the role of DDR2 mutations as predictive biomarkers in HNSCC are warranted. While SFK inhibitors are FDA approved in several hematological malignancies, their role has not been defined in solid tumors and is the subject of ongoing preclinical and clinical investigations (61). A 12-patient, single-arm, phase II study of dasatinib in unselected patients with recurrent/metastatic HNSCC failed to demonstrate significant activity; however, the DDR2 status of patients' tumors was unknown (62). A biomarker-guided trial in which patients with DDR2-mutated tumors are selected to receive a SFK inhibitor has not been performed. A variety of sequencing platforms in common clinical use are capable of rapidly assessing the mutational status of DDR2 and other genes in HNSCC tumor samples (63). The implementation of such techniques will be required to determine to what degree, if any, DDR2 mutations are enriched in metastatic/recurrent disease overall and to correlate mutational status with treatment response and outcomes.

Limitations of the present study include the relatively small sample size, the use of a cohort of convenience where adequate biologic material was available for sequencing, a lack of distant metastases, variations in tumor sample purity, and the assessment of a single sample per tumor, thereby precluding assessment of intratumor heterogeneity. While these findings will require validation in future, larger sequencing studies, we believe this is the first mutational analysis of patient-matched tumor pairs in the setting of synchronous nodal metastasis and metachronous recurrence in HNSCC. The findings provide an opportunity to guide future investigations.

\section{Methods}

Patient selection and DNA extraction. Fresh-frozen tumor pairs and matched normal tissue were banked in the University of Pittsburgh head and neck tissue bank and made available for sequencing in this project under the auspices of an observational research study funded by the Specialized Program of Research Excellence (SPORE) as previously described (29). Genomic DNA for WES was extracted from whole blood and fresh-frozen tumor tissue using the DNeasy Blood \& Tissue Kit (QIAGEN) according to the manufacturer's instructions.

WES and analysis. Targeted capture was performed using the NimbleGen 2.1 Exome reagent, followed by sequencing on the Illumina platform, and downstream was processing performed as previously described $(64,65)$. Briefly, sequences were aligned to NCBI Build 37 of the human genome using the ELAND program (Illumina). Somatic mutations were called based on the significance of differences in reference and nonreference read distributions between tumor and matched normal samples. Under the assumption that few if any clonal somatic changes should occur in blood DNA, as a control the same test was applied to germline DNA using tumor DNA as reference, which, as previously shown, yields high validation rates of putative somatic mutations (66). If a mutation was called in either tumor of a patient-matched pair, it was considered present in the second tumor if there were at least 5 independent reads supporting the variant call. Calls were further evaluated by manual inspection of read alignments. Sequencing data were deposited in the NCBI's database of Genotypes and Phenotypes (dbGaP phs0001007.v1.p1).
Additional tissue samples from 6 tumors (the primary tumors of patients PY-1, PY-7, PY-13, PY-19, PY-24, and the recurrent tumor from patient PY-3) underwent WES under the auspices of other studies (29, 30). The alternative informatics pipeline and sequencing methods are described in the published reports. In these 6 tumors, $78 \%$ of the mutations detected by the sequencing and bioinformatics pipeline in this study were also identified in the other studies (Supplemental Table 9). A lack of hotspot mutations in PIK3CA (AAs 542, 545, and 1047) was confirmed by Sanger sequencing of the tumor samples used in this study (data not shown).

MutSigCV (v1.3.01) was used to determine whether genes were mutated more often than expected by chance (33). CNVs were identified by comparing coverage depth ratios of tumor and matched normal samples after normalizing for mean coverage depth of each exome and changes in minor allele frequency at informative SNPs. GISTIC2.0 was used to assess the significance of recurrent CNVs, and the PyClone algorithm was used to assess clonality, as described previously $(36,37)$.

Cell cultures. The HNSCC cell lines BICR 18 and PE/CA-PJ34 (clone C12) were obtained from Sigma-Aldrich; UPCI 15B was a gift from Theresa Whiteside (University of Pittsburgh Cancer Institute). The HNSCC cell lines were grown in the following culture mediums, each containing $10 \%$ fetal calf serum and $1 \times$ penicillin/ streptomycin solution (Invitrogen): UPCI 15B in DMEM, BICR 18 in DMEM with $2 \mathrm{mM}$ glutamine (Mediatech Inc.) and $0.4 \mu \mathrm{g} / \mathrm{ml}$ hydrocortisone, and the PE/CA-PJ34 (clone C12) cells in Iscove's Modifed Dulbecco Minimum Essential Medium with $2 \mathrm{mM}$ glutamine (Mediatech Inc.). All cell lines were maintained in a humidified cell incubator at $37^{\circ} \mathrm{C}, 5 \% \mathrm{CO}_{2}$.

Drug treatment and survival assays. HNSCC cells were plated at the indicated concentrations in 48-well culture plates overnight and treated with the indicated concentrations of dasatinib (Bristol-Myers Squibb) for 48 hours, at which point MTT (Sigma-Aldrich) was performed according to the manufacturer's instructions, as previously described (26). Growth curves were generated using GraphPad Prism 6 software as outlined in Statistics.

Invasion assays. Invasion of BICR 18 cells in the presence or absence of dasatinib was tested using BioCoat migration and Matrigel-coated invasion chambers (BD Biosciences) according to the manufacturer's instructions. Briefly, $2.0 \times 10^{4} \mathrm{BICR} 18$ cells were placed in migration (uncoated) chambers or invasion (Matrigel coated) chambers. They were treated with DMSO $(n=2)$ or the indicated doses of dasatinib $(n=3)$ for 24 hours. The experiment was repeated 3 times, and cells were counted and averaged from 4 photomicrographs from each membrane. Bar graphs and statistical analysis generated in GraphPad Prism 6 are outlined in Statistics.

DDR2 knockdown. BICR 18 cells were plated at $10 \%$ confluence in 96-well plates overnight, treated with $8 \mu \mathrm{l}$ polybrene, and infected with $15 \mu \mathrm{l}$ of MISSION pLKO.1-puro lentiviral particles containing control or $\alpha-D D R 2$ shRNA constructs ( $\alpha$-EGFP control, 121117 5'-CCGGCCCATGCCTATGCCACTCCATCTCGAGATGGAGTGGCATAGGCATGGGTTTTTG-3'， or 195105 5'-CCGGCGAAACTGTTTAGTGGGTAAGCTCGAGCTTACCCACTAAACAGTTTCGTTT-TTTG-3') (Sigma-Aldrich). Seventy-two hours later, cells were split into 96-well plates and cultured for 2 weeks in media containing puromycin to derive subclones expressing the shRNA constructs. Cells were then cultured in full medium without puromycin until sufficient volumes were obtained for phenotypic analyses (3-6 weeks). 
Cloning and mutagenesis. WT EGFP was cloned into the retroviral vector pMXs-puro (Cell Biolabs Inc.). WT DDR2 cloned into a pWZLBlast vector was a gift from Peter Hammerman (Dana-Farber/Harvard Cancer Center, Boston, Massachusetts, USA) and was used as a template for site-directed mutagenesis using the QuikChange SiteDirected Mutagenesis Kit (Stratagene) (49). Mutagenesis of the DDR2 WT gene was performed according to the manufacturer's instructions and confirmed by Sanger sequencing.

Mutant DDR2 expression. Retroviruses were generated using the Platinum Retrovirus Expression Systems (Cell Biolabs), Fugene HD (Promega), and retroviral vectors carrying the gene of interest (pMXs-puro-EGFP as control, pWZL-Blast-DDR2 [WT], pWZL-Blast$D D R 2$ [mutants]) according to the manufacturers' instructions, as previously published (26).

Western blotting. Standard Western blotting was performed as previously described (67). Primary antibodies were as follows: $\alpha$-DDR2 (catalog 12133, Cell Signaling Technology Inc.), $\alpha$ - $\beta$-tubulin (ab6046, Abcam), goat anti-rabbit IgG (H+L)-HRP-conjugated secondary antibodies (catalog 170-6515, Bio-Rad), and Luminol reagent (sc-2048, Santa Cruz Biotechnology Inc.).

Statistics. Mean values with SEM from replicate experiments plated as indicated in figure legends were plotted as mean values \pm SEM in Graphpad Prism 6. A sigmoidal dose-response curve with automatic outlier elimination was applied to generate optimized data sets. The finalized data sets, with outliers removed, were used to generate growth curves using the least squares fit log (inhibitor) versus normalized response-variable slope equation. The $\log \mathrm{IC}_{50}$ values were compared using an extra sum of the squares $\mathrm{F}$ test (null hypothesis indicates the $\log \mathrm{IC}_{50}$ values are identical; the null hypothesis was rejected in cases where $P<0.05)$. For bar graphs, mean values with SEM were calculated and plotted as above and compared using an unpaired, 2-tailed $t$ test with Welch's correction.

Study approval. The patient cohort consisted of HNSCC patients treated at the University of Pittsburgh Medical Center otolaryngol- ogy clinics who were enrolled in an observational research study supported by the University of Pittsburgh's SPORE in head and neck cancer. All participants provided written informed consent, and the study was approved by the University of Pittsburgh Institutional Review Board.

\section{Author contributions}

Conception and design were by MLH, JRG, SIC, and RPL. Development of methodology was performed by MLH. Acquisition of data was performed by MLH, GG, BBD, YZ, MLF, VWYL, SIC, and LW. Analysis and interpretation of data were performed by $\mathrm{MLH}$, GG, JEB, BBD, WEG, JRG, VWYL, RPL, and RSH. Writing, review, and/or revision of the manuscript were done by MLH, GG, JEB, BBD, JRG, RPL, and RSH. Administrative, technical, or material support was provided by MLH, JRG, SIC, and LW.

\section{Acknowledgments}

We would like to thank Theresa Whiteside for graciously providing the UPCI 15B cell line and Peter Hammerman for graciously providing the DDR2 retroviral expression construct. This work was supported by grants from the NCI (1F30CA180235-01A1 to M.L. Hedberg, and P50CA097190 to J.R. Grandis), the Agency for Science, Technology and Research of Singapore (fellowship to G. Goh), the American Cancer Society (CRP-08-229-01 to J.R. Grandis), and Gilead Sciences Inc. (research support to R.P. Lifton and R.S. Herbst).

Address correspondence to: Richard Lifton, Department of Genetics, P.O. Box 208005, 333 Cedar Street, New Haven, Connecticut 06520-8005, USA. Phone: 203.737.4420; E-mail: richard.lifton@ yale.edu. Or to: Jennifer R. Grandis, University of California, San Francisco, Box 0558, 550-16th Street, 6th Floor, San Francisco, California 94143, USA. Phone: 415.514.8084; E-mail: jennifer. grandis@ucsf.edu.
1. Ferlay J, et al. GLOBOCAN 2012 v1.0, Cancer Incidence and Mortality Worldwide: IARC CancerBase No. 11. Lyon, France: International Agency for Research on Cancer; 2013. WHO Web site. http://globocan.iarc.fr. Accessed November 3, 2015.

2. Marur S, D'Souza G, Westra WH, Forastiere AA. HPV-associated head and neck cancer: a virus-related cancer epidemic. Lancet Oncol. 2010;11(8):781-789.

3. Leemans CR, Braakhuis BJ, Brakenhoff RH. The molecular biology of head and neck cancer. Nat Rev Cancer. 2011;11(1):9-22.

4. Roepman P, de Jager A, Groot Koerkamp MJ, Kummer JA, Slootweg PJ, Holstege FC. Maintenance of head and neck tumor gene expression profiles upon lymph node metastasis. Cancer Res. 2006;66(23):11110-11114.

5. National Cancer Institute. Surveillance, Epidemiology, and End Results (SEER) Program. SEER ${ }^{*}$ Stat Databases: November 2013 Submission. NIH Web site. http://seer.cancer.gov/data/ seerstat/nov2013/. Updated November 5, 2014. Accessed November 3, 2015.

6. de Juan J, et al. Inclusion of extracapsular spread in the pTNM classification system: a proposal for patients with head and neck carcinoma. JAMA Otolaryngol Head Neck Surg. 2013;139(5):483-488.

7. Wan XC, Egloff AM, Johnson J. Histological assessment of cervical lymph node identifies patients with head and neck squamous cell carcinoma (HNSCC): who would benefit from chemoradiation after surgery? Laryngoscope. 2012;122(12):2712-2722.

8. Ho AS, Kraus DH, Ganly I, Lee NY, Shah JP, Morris LG. Decision making in the management of recurrent head and neck cancer. Head Neck. 2014;36(1):144-151.

9. Graveland AP, et al. Molecular diagnosis of minimal residual disease in head and neck cancer patients. Cell Oncol (Dordr). 2012;35(5):367-375.

10. León X, et al. Second, third, and fourth head and neck tumors. A progressive decrease in survival. Head Neck. 2012;34(12):1716-1719.

11. Burrell RA, McGranahan N, Bartek J, Swanton $\mathrm{C}$. The causes and consequences of genetic heterogeneity in cancer evolution. Nature. 2013;501(7467):338-345.

12. Gerlinger $\mathrm{M}$, et al. Intratumor heterogeneity and branched evolution revealed by multiregion sequencing. N Engl J Med. 2012;366(10):883-892.

13. Ding L, et al. Clonal evolution in relapsed acute myeloid leukaemia revealed by whole-genome sequencing. Nature. 2012;481(7382):506-510.

14. Mullighan CG, et al. Genomic analysis of the clonal origins of relapsed acute lymphoblastic leukemia. Science. 2008;322(5906):1377-1380.

15 . Wagle $\mathrm{N}$, et al. MAP kinase pathway alterations in BRAF-mutant melanoma patients with acquired resistance to combined RAF/MEK inhibition. Cancer Discov. 2014;4(1):61-68.

16. André F, et al. Comparative genomic hybridisation array and DNA sequencing to direct treatment of metastatic breast cancer: a multicentre, prospective trial (SAFIR01/UNICANCER). Lancet Oncol. 2014;15(3):267-274.

17. Johnson BE, et al. Mutational analysis reveals the origin and therapy-driven evolution of recurrent glioma. Science. 2014;343(6167):189-193.

18. Kreso A, et al. Variable clonal repopulation dynamics influence chemotherapy response in colorectal cancer. Science. 2013;339(6119):543-548.

19. Yu M, et al. Cancer therapy. Ex vivo culture of circulating breast tumor cells for individualized testing of drug susceptibility. Science. 
2014;345(6193):216-220.

20. Cromer A, et al. Identification of genes associated with tumorigenesis and metastatic potential of hypopharyngeal cancer by microarray analysis. Oncogene. 2004;23(14):2484-2498.

21. Ginos MA, et al. Identification of a gene expression signature associated with recurrent disease in squamous cell carcinoma of the head and neck. Cancer Res. 2004;64(1):55-63.

22. Reis PP, et al. A gene signature in histologically normal surgical margins is predictive of oral carcinoma recurrence. BMC Cancer. 2011;11:437.

23. van Hooff SR, et al. Validation of a gene expression signature for assessment of lymph node metastasis in oral squamous cell carcinoma. JClin Oncol. 2012;30(33):4104-4110.

24. Agrawal N, et al. Exome sequencing of head and neck squamous cell carcinoma reveals inactivating mutations in NOTCH1. Science. 2011;333(6046):1154-1157.

25. India Project Team of the International Cancer Genome Consortium. Mutational landscape of gingivo-buccal oral squamous cell carcinoma reveals new recurrently-mutated genes and molecular subgroups. Nat Commun. 2013;4:2873.

26. Lui VW, et al. Frequent mutation of the PI3K pathway in head and neck cancer defines predictive biomarkers. Cancer Discov. 2013;3(7):761-769.

27. Lui VW, et al. Frequent mutation of receptor protein tyrosine phosphatases provides a mechanism for STAT3 hyperactivation in head and neck cancer. Proc Natl Acad Sci U S A. 2014;111(3):1114-1119.

28. Pickering CR, et al. Integrative genomic characterization of oral squamous cell carcinoma identifies frequent somatic drivers. Cancer Discov. 2013;3(7):770-781.

29. Stransky N, et al. The mutational landscape of head and neck squamous cell carcinoma. Science. 2011;333(6046):1157-1160.

30. Cancer Genome Atlas Network. Comprehensive genomic characterization of head and neck squamous cell carcinomas. Nature. 2015;517(7536):576-582.

31. Seiwert TY, et al. Integrative and comparative genomic analysis of HPV-positive and HPV-negative head and neck squamous cell carcinomas. Clin Cancer Res. 2015;21(3):632-641.

32. Edge S, Byrd DR, Compton CC, Fritz AG, Greene FL, Trotti A, eds. AJCC Cancer Staging Manual. Vol. 649. New York, New York, USA: Springer; 2010.

33. Lawrence MS, et al. Mutational heterogeneity in cancer and the search for new cancer-associated genes. Nature. 2013;499(7457):214-218.

34. Cancer Genome Atlas Research Network, et al. The Cancer Genome Atlas Pan-Cancer analysis project. Nat Genet. 2013;45(10):1113-1120.

35. Vogelstein B, Papadopoulos N, Velculescu VE, Zhou S, Diaz LA Jr, Kinzler KW. Cancer genome landscapes. Science. 2013;339(6127):1546-1558.

36. Mermel CH, Schumacher SE, Hill B, Meyerson ML, Beroukhim R, Getz G. GISTIC2.0 facilitates sensitive and confident localization of the targets of focal somatic copy-number alteration in human cancers. Genome Biol. 2011;12(4):R41.

37. Roth A, et al. PyClone: statistical inference of clonal population structure in cancer. Nat Methods. 2014;11(4):396-398.

38. Maranto AR. Primary structure, ligand binding, and localization of the human type 3 inositol 1,4,5-trisphosphate receptor expressed in intestinal epithelium. J Biol Chem. 1994;269(2):1222-1230.

39. Srivastava M1, Eidelman O, Leighton X, Glasman M, Goping G, Pollard HB. Anx7 is required for nutritional control of gene expression in mouse pancreatic islets of Langerhans. Mol Med. 2002;8(12):781-797.

40. Sakakura C, et al. Possible involvement of inositol 1,4,5-trisphosphate receptor type 3 (IP3R3) in the peritoneal dissemination of gastric cancers. Anticancer Res. 2003;23(5A):3691-3697.

41. Shibao K, et al. The type III inositol 1,4,5-trisphosphate receptor is associated with aggressiveness of colorectal carcinoma. Cell Calcium. 2010;48(6):315-323.

42. Mound A, Rodat-Despoix L, Bougarn S, OuadidAhidouch H, Matifat F. Molecular interaction and functional coupling between type 3 inositol 1,4,5-trisphosphate receptor and BKCa channel stimulate breast cancer cell proliferation. Eur J Cancer. 2013;49(17):3738-3751.

43. Zhang Y, et al. An essential role of discoidin domain receptor 2 (DDR2) in osteoblast differentiation and chondrocyte maturation via modulation of Runx2 activation. J Bone Miner Res. 2011;26(3):604-617.

44. Walsh LA, Nawshad A, Medici D. Discoidin domain receptor 2 is a critical regulator of epithelial-mesenchymal transition. Matrix Biol. 2011;30(4):243-247.

45. Leitinger B. Discoidin domain receptor functions in physiological and pathological conditions. Int Rev Cell Mol Biol. 2014;310:39-87.

46. Yan Z, et al. Discoidin domain receptor 2 facilitates prostate cancer bone metastasis via regulating parathyroid hormone-related protein. Biochim Biophys Acta. 2014;1842(9):1350-1363.

47. Zhang K, et al. The collagen receptor discoidin domain receptor 2 stabilizes SNAIL1 to facilitate breast cancer metastasis. Nat Cell Biol. 2013;15(6):677-687.

48. Xu J, et al. Overexpression of DDR2 contributes to cell invasion and migration in head and neck squamous cell carcinoma. Cancer Biol Ther. 2014;15(5):612-622.

49. Hammerman PS, et al. Mutations in the DDR2 kinase gene identify a novel therapeutic target in squamous cell lung cancer. Cancer Discov. 2011;1(1):78-89.

50. Beauchamp EM, et al. Acquired resistance to dasatinib in lung cancer cell lines conferred by DDR2 gatekeeper mutation and NF1 loss. Mol Cancer Ther. 2014;13(2):475-482.

51. Pitini V, Arrigo C, Di Mirto C, Mondello P, Altavilla $G$. Response to dasatinib in a patient with
SQCC of the lung harboring a discoid-receptor-2 and synchronous chronic myelogenous leukemia. Lung Cancer. 2013;82(1):171-172.

52. Barretina J, et al. The Cancer Cell Line Encyclopedia enables predictive modelling of anticancer drug sensitivity. Nature. 2012;483(7391):603-607.

53. Zhang XC, et al. Tumor evolution and intratumor heterogeneity of an oropharyngeal squamous cell carcinoma revealed by whole-genome sequencing. Neoplasia. 2013;15(12):1371-1378.

54. Slaughter DP, Southwick HW, Smejkal W. Field cancerization in oral stratified squamous epithelium; clinical implications of multicentric origin. Cancer. 1953;6(5):963-968.

55. Tabatabaeifar S, Kruse TA, Thomassen M, Larsen MJ, Sørensen JA. Use of next generation sequencing in head and neck squamous cell carcinomas: a review. Oral Oncol. 2014;50(11):1035-1040.

56. Iwai LK, et al. Phosphoproteomics of collagen receptor networks reveals SHP-2 phosphorylation downstream of wild-type DDR2 and its lung cancer mutants. Biochem J. 2013;454(3):501-513.

57. Wang K, et al. Exome sequencing identifies frequent mutation of ARID1A in molecular subtypes of gastric cancer. Nat Genet. 2011;43(12):1219-1223.

58. Cancer Genome Atlas Research Network, et al. Integrated genomic characterization of endometrial carcinoma. Nature. 2013;497(7447):67-73.

59. Cerami E, et al. The cBio cancer genomics portal: an open platform for exploring multidimensional cancer genomics data. Cancer Discov. 2012;2(5):401-404.

60. Forbes SA, et al. COSMIC (the Catalogue of Somatic Mutations in Cancer): a resource to investigate acquired mutations in human cancer. Nucleic Acids Res. 2010;38(Database issue):D652-D657.

61. Lindauer M, Hochhaus A. Dasatinib. Recent Results Cancer Res. 2014;201:27-65.

62. Brooks HD, et al. Phase 2 study of dasatinib in the treatment of head and neck squamous cell carcinoma. Cancer. 2011;117(10):2112-2119.

63. MacConaill LE, et al. Prospective enterpriselevel molecular genotyping of a cohort of cancer patients. JMol Diagn. 2014;16(6):660-672.

64. Goh G, et al. Recurrent activating mutation in PRKACA in cortisol-producing adrenal tumors. Nat Genet. 2014;46(6):613-617.

65. Scholl UI, et al. Somatic and germline CACNA1D calcium channel mutations in aldosterone-producing adenomas and primary aldosteronism. Nat Genet. 2013;45(9):1050-1054.

66. Choi $\mathrm{M}$, et al. $\mathrm{K}^{+}$channel mutations in adrenal aldosterone-producing adenomas and hereditary hypertension. Science. 2011;331(6018):768-772.

67. Zhang $Q$, et al. Antitumor mechanisms of combined gastrin-releasing peptide receptor and epidermal growth factor receptor targeting in head and neck cancer. Mol Cancer Ther. 2007;6(4):1414-1424 